\title{
Stockage intersaisonnier de chaleur par conduction Bilan de deux années d'expérimentation
}

\author{
Interseasonal heat storage by conduction \\ Record of two years of experiments
}

\author{
G. Leroy \\ Laboratoire de Thermocinétique, \\ Université de Nantes
}

\author{
R. Sanejouand
}

Laboratoire central des Ponts et Chaussées

Centre de Nantes
Le système considéré est constitué d'un réseau d'échangeurs tubulaires, verticaux, implantés par forage dans un sol peu perméable qui sert de matériau de stockage. Il fonctionne par conduction à un niveau de température compatible avec une utilisation directe de l'énergie déstockée.

Un stock expérimental défini sur la base d'une modélisation en régime périodique établi est présenté.

Certaines phases de l'évolution thermique sont interprétées en vue de préciser les phénomènes qui régissent les transferts de chaleur dans le stock et accéder aux caractéristiques thermocinétiques apparentes du sol. Les valeurs obtenues sont confrontées aux mesures effectuées avec une sonde à choc thermique.

L'évolution du régime hydrique dans la couche superficielle non saturée, siège de gradients thermiques importants, est comparée à celle observée en site libre.
The system considered consists of a network of vertical tube heat exchangers installed by drilling in a soil with $a$ low permeability used as storage material. It operates by conduction at a temperature level compatible with direct use of the destored energy.

An experimental storage defined on the basis of an established periodic model is described.

Certain phases of the thermal variations are interpreted to refine the phenomena determining heat transfers in the storage material and access the apparent thermokinetic characteristics of the soil. The values obtained are compared with measurements made with a thermal shock probe.

The variation of the hydric condition of the unsaturated surface layer, where considerable thermal gradients occur, is compared with that observed in a free site.

\section{Introduction}

Le principe de stockage intersaisonnier de chaleur dans le sol, à l'aide d'échangeurs tubulaires placés dans des forages, est maintenant bien connu. Les transferts thermiques au sein du milieu se font par conduction. Le procédé convient aux sols fins peu perméables ou massifs, largement répandus. Pour une emprise en surface réduite, des volumes de sol très importants peuvent être ainsi mis en œuvre et les énergies transférées deviennent alors considérables.

Dans la région nantaise, le Laboratoire de thermocinétique de l'Université de Nantes et le Laboratoire central des Ponts et Chaussées de Bouguenais ont engagé, en 1981, une action visant à établir la faisabilité théorique et expérimentale de ce procédé de stockage, fonctionnant de plus à un niveau de température compatible avec une utilisation directe de l'énergie déstockée.
Au plan théorique, les modèles de calcul développés [1 à 3] ont permis d'approcher les performances d'une unité de stockage et de dégager les mécanismes qui régissent les transferts de chaleur en régime périodique établi. En particulier le rôle essentiel du niveau thermique de la puissance échangée et de la distance entre échangeurs a été clairement montré.

Sur la base des résultats de cette étude, un stock expérimental de taille significative a été réalisé avec l'aide du C.O.M.E.S. Equipé bien évidemment de capteurs thermiques mais aussi de capteurs hydriques, ce pilote devait servir en premier lieu à observer le comportement du milieu de stockage et à mettre en évidence d'éventuels phénomènes parasites tels que des circulations d'eau localisées ou des transferts de masse en phase vapeur dus aux gradients thermiques dans la zone superficielle non 
saturée. Il devait servir ensuite à caler les modèles retenus pour décrire les transferts de chaleur en régime transitoire.

L'expérimentation a débuté au mois de juin 1982 et a duré deux ans. L'objet de cet article est de présenter une synthèse des principaux résultats expérimentaux obtenus au cours de ces deux années.

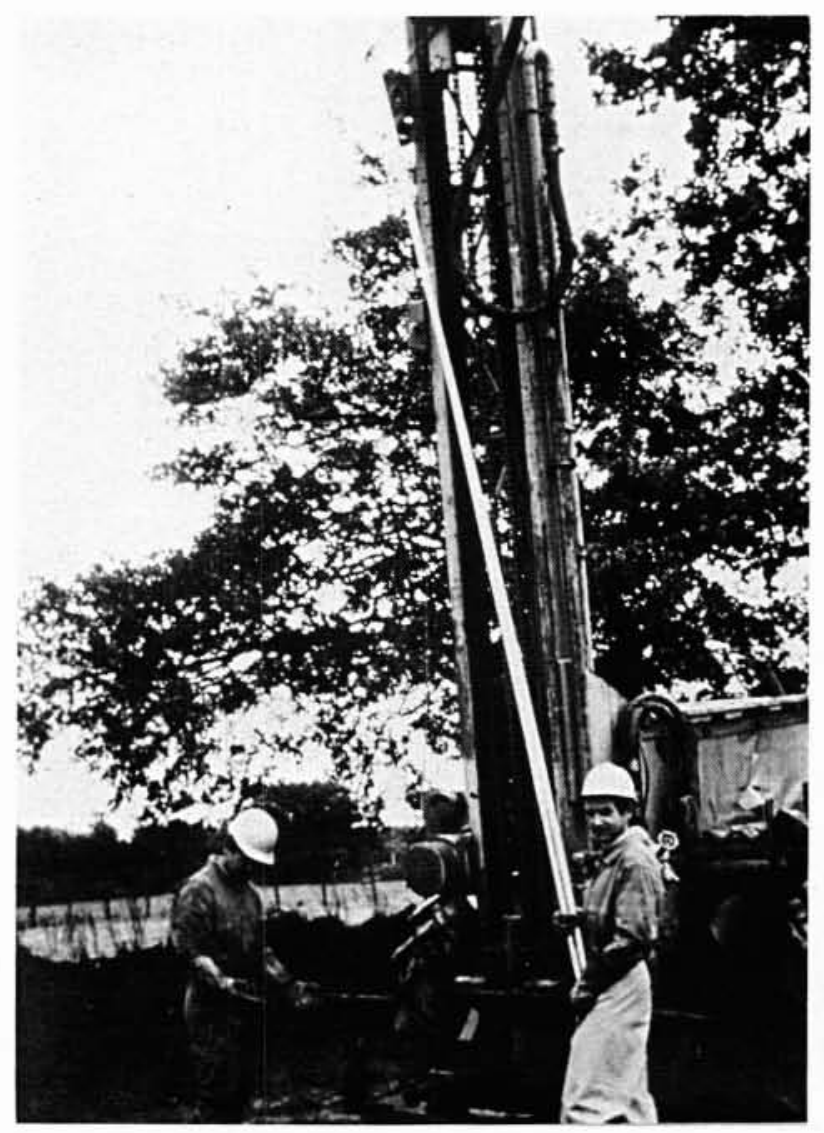

Figure 1. - Mise en place d'un échangeur.

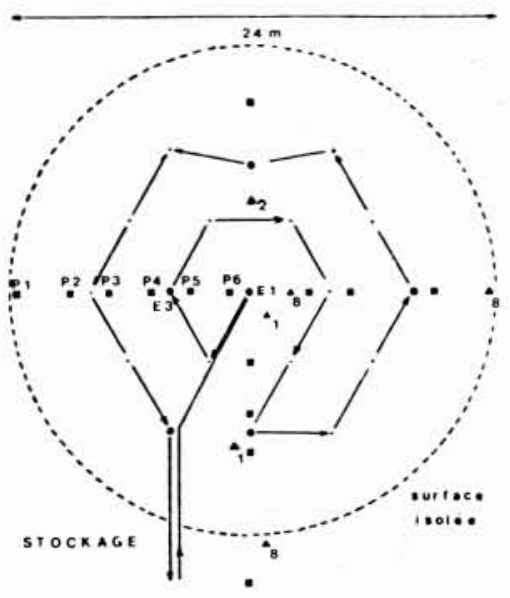

SOL $11.5,12,18 \mathrm{~m})$

- temperatuags entree-sortie oun echangeur

a SONDE de conductivite (1,2 ou a m

Figure 2. - Instrumentation thermique.
Après avoir décrit la réalisation du stock expérimental (site, système de stockage, instrumentation) et le programme expérimental, on s'attachera successivement aux mesures thermiques et plus particulièrement à leur interprétation au sens de la caractérisation thermocinétique du milieu de stockage, puis aux mesures hydriques.

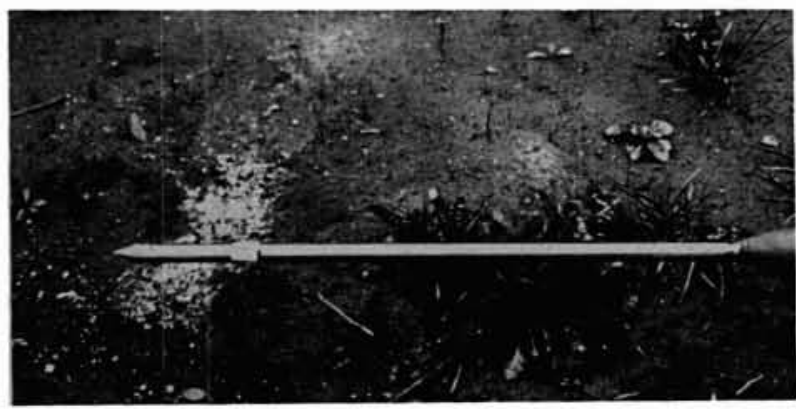

Figure 4. - Sonde de mesure de la conductivité et de la diffusivité thermique du sol.

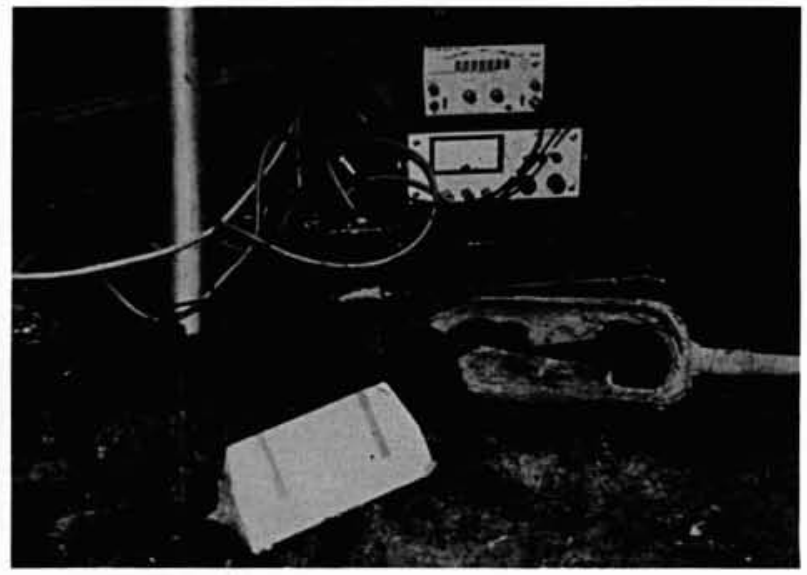

Figure 5. - Cellule capacitive.
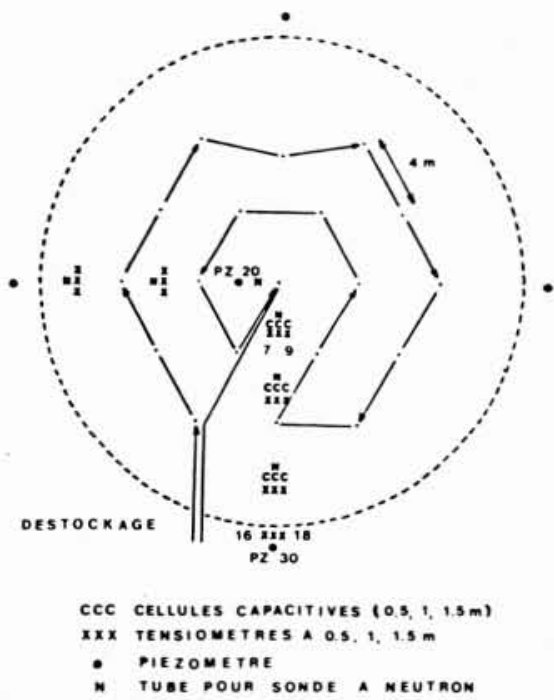

Figure 3. - Instrumentation hydrique. 


\section{Réalisation du stock expérimental}

\subsection{Site retenu}

Le stock a été réalisé sur le terrain du L.C.P.C. à Bouguenais près de Nantes. Les conditions géologiques et hydrogéologiques bien connues, y sont à priori favorables. La surface du sol est sensiblement horizontale. Le sol est composé de schistes altérés. La nappe phréatique, pratiquement affleurante en hiver, descend l'été jusqu'à environ trois mètres de profondeur, ces mouvements étant dus essentiellement à l'infiltration des précipitations et à l'évapotranspiration. La perméabilité du sol et le gradient hydraulique transversal sont très faibles.

\section{I.2. Description du système de stockage}

La structure d'échange comprend dix-neuf "épingles " (figure 1) de $15 \mathrm{~m}$ de long réalisées en tube d'acier galvanisé (ø 21-27 mm) mises en place dans des forages remplis de bentonite selon une maille triangulaire de $4 \mathrm{~m}$ et réparties en deux couronnes hexagonales concentriques autour de l'échangeur central. Le "volume" du stock, c'est-à-dire la zone " active " de sol participant aux cycles de stockage-déstockage peut être estimé à environ $5000 \mathrm{~m}^{3}$. Les échangeurs sont reliés en série. En période de stockage, le fluide caloporteur, de l'eau, circule de l'échangeur central vers la périphérie, inversement en période de déstockage, permettant ainsi de réduire les pertes thermiques dans le massif.

Pour le stockage, l'eau est chauffée électriquement dans une cuve isolée, d'environ un mètre cube avec des thermoplongeurs d'une puissance maximale de $36 \mathrm{~kW}$. Dans ce cas, la circulation du fluide est assurée par une pompe volumétrique à débit variable de 0 à $1,5 \mathrm{~m}^{3} / \mathrm{h}$. Pour le déstockage, le circuit fluide est directement couplé au réseau urbain de distribution d'eau.

La surface du sol, pour la partie "active " est isolée avec une couche de paille d'épaisseur $1 \mathrm{~m}$ placée entre deux films de polyéthylène entraînant aussi une imperméabilisation de cette zone.

\section{I.3. Instrumentation}

Les répartitions des différents capteurs thermiques et hydriques sont données respectivement sur les figures 2 et 3.

\section{Le suivi de l'état thermique porte sur:}

- Les températures d'entrée et de sortie de l'eau sur 7 des 19 échangeurs dont le premier et le dernier; ces températures associées au débit d'eau mesuré à partir d'un compteur volumétrique permettent de connaître les puissances échangées localement et au niveau de l'installation globale ainsi que le niveau thermique des échanges.

- La température dans le sol au moyen de 14 profils verticaux (à $1 \mathrm{~m}, 5 \mathrm{~m}, 12 \mathrm{~m}$ et $18 \mathrm{~m}$ de profondeur) implantés sur deux diamètres.

- La mesure de la conductivité et de la diffusivité thermique à l'aide de six sondes (figure 4) mises en place à diverses profondeurs (trois à $8 \mathrm{~m}$, une à $2 \mathrm{~m}$ et deux à $1 \mathrm{~m})$.

\section{Le suivi de l'état hydrique est assuré par :}

- Cinq piézomètres dont quatre en périphérie et un central.

- Six tubes pour réaliser des profils de teneur en eau jusqu'à $2 \mathrm{~m}$ de profondeur à l'aide d'une sonde neutronique. Ces profils sont complétés par des mesures effectuées avec six séries de trois cellules capacitives (figure 5) situées à $0.5 \mathrm{~m}, 1 \mathrm{~m}$ et $1.5 \mathrm{~m}$ de profondeur.

- Six séries de trois tensiomètres placés à $0.5 \mathrm{~m}, 1 \mathrm{~m}$ et $1.5 \mathrm{~m}$ de la surface.

Ces capteurs sont situés eux aussi sur deux diamètres perpendiculaires du stock.

\section{Programme et suivi expérimental}

Le programme de chargement du stock et d'extraction de la chaleur est très simple.

L'injection de chaleur se fait par paliers de puissance constante, le débit de circulation étant choisi pour que la température reste inférieure à $70^{\circ} \mathrm{C}$.

Pendant la première phase de stockage qui a duré 4,5 mois (du 15/06/82 au 2/11/83), $60000 \mathrm{kw} / \mathrm{h}$ ont été injectés. La température au cœur du stock, atteinte en fin de stockage, étant trop basse $35^{\circ} \mathrm{C}$, aucun déstockage n'a été entrepris au cours de l'hiver qui a suivi (6 mois). La deuxième phase de stockage, un peu plus longue, environ six mois et demi, s'est déroulée en deux temps : du $03 / 05 / 83$ au $26 / 09 / 83$ et du $28 / 11 / 83$ au $13 / 01 / 84$, ceci en raison d'une panne intervenue sur la pompe de circulation. Le centre du stock a été ainsi amené à près de $43^{\circ} \mathrm{C}$ et $80000 \mathrm{kw} / \mathrm{h}$ ont été stockés.

Au cours des deux mois et demi suivants, le stock n'a été soumis qu'aux conditions naturelles. Cette phase de refroidissement permet de compléter les observations faites d'une part, au cours de la première relaxation hivernale $(2 / 11 / 82$ au $3 / 05 / 83)$, le niveau de température étant plus élevé, d'autre part lors de la relaxation survenue à l'automne 83 , le sol étant saturé.

Le déstockage s'est déroulé du 21/03/84 au 13/06/84. Près de $11000 \mathrm{kw} / \mathrm{h}$ ont été ainsi restitués à un niveau de température compris entre 20 et $30^{\circ} \mathrm{C}$. Cette phase a été 
mise à profit pour étudier la dynamique du stock en réponse à différentes sollicitations, débit d'injection du fluide caloporteur variable, extractions de courtes durées (quelques heures) puis extraction continue.

L'acquisition des données thermiques a été automatisée. Pour les phases de stockage, la période de scrutation des capteurs placés sur les échangeurs a été fixée à une heure et celle des thermocouples dans le sol à huit heures. Au cours des relaxations hivernales, deux scrutations quotidiennes pour l'ensemble des capteurs se sont avérées amplement suffisantes.

Les informations fournies par l'instrumentation hydrique ont été recueillies manuellement : tensiomètres et cellules capacitives étant relevés deux fois par jour, puis une seule fois, les mesures de teneur en eau par méthode neutronique étant effectuées une fois par semaine puis une fois tous les quinze jours.

\section{Mesures thermiques}

Le traitement de l'ensemble des données et notamment les données correspondant aux phases d'injection ou d'extraction de la chaleur nécessite l'élaboration d'un modèle numérique assez lourd. Ce modèle doit en effet prendre en compte des phénomènes couplés tels que la conduction dans le solide et la convection au niveau du fluide caloporteur, mais aussi les singularités liées à la présence de nombreux échangeurs. La réalisation forcément longue d'un tel code de calcul est en cours. En l'absence des résultats de cette modélisation, on peut cependant tirer, de la seule observation de ces données, certains enseignements. Par ailleurs, le début de la première phase d'injection de la chaleur et les phases de relaxation naturelle se prêtent aisément à des interprétations visant à caractériser le comportement thermocinétique du milieu de stockage. Ces analyses conduisent à la détermination locale ou à l'échelle du stockage, de la conductivité et de la diffusivité thermique du milieu, grandeurs qu'il est intéressant de comparer aux mesures très ponctuelles obtenues avec les sondes.

\subsection{Observation d'ensemble de quelques évolutions}

A titre d'illustration, les évolutions de la température d'entrée de l'échangeur central et du dernier échangeur pendant les phases "actives" sont représentées sur la figure 6. Le programme de chauffe et la puissance soutirée au déstockage y sont aussi indiqués ainsi que les incidents venus perturber ce programme. La figure 7 donne les évolutions de quelques températures dans le sol selon un rayon à cinq mètres de profondeur aux droits des profils P1, P2, P4 et P6. Quelques commentaires immédiats peuvent être faits sur cette figure :

- au cours de la seconde année, le stock est nettement plus chaud. Son niveau thermique moyen s'élève progressivement comme en témoigne l'évolution de température du capteur du profil $\mathrm{Pl}$, situé à la frontière du stock, à $3 \mathrm{~m}$ du plus proche échangeur;

- malgré une puissance extraite non négligeable, à partir de mars 84 les courbes de température s'infléchissent peu, même au voisinage des échangeurs E1 et E3. Les pertes thermiques sont prépondérantes en raison de la petite taille du stock et du niveau de température.

L'observation attentive de l'ensemble des évolutions et plus particulièrement des phases de refroidissement natu- rel qui offrent une image plus homogène du champ de température ne permet pas de mettre en évidence d'anomalie (écoulement transversal...) en regard du fonctionnement supposé du système de stockage.

\subsection{Interprétation des températures dans le sol et sur les échangeurs}

Au début de la première phase d'injection de la chaleur, l'évolution du champ de température au voisinage d'un échangeur n'est pas influencée par la présence des autres échangeurs. Il est facile, dans ce cas, de relier cette évolution de température $\theta$ à la puissance dissipée, moyennant les hypothèses suivantes :

- le milieu est initialement isotherme. Il est infini, homogène et isotrope.

- La puissance est dissipée de façon uniforme le long de l'échangeur schématisé par une source linéaire de chaleur et le phénomène thermique est purement radial.

$\theta$ a alors pour expression :

$$
\theta(r, t)=\frac{1}{4 \pi \lambda} \frac{d p}{d t} * E_{1}\left(\frac{r^{2}}{4 a t}\right)
$$

avec $\quad r$ la distance de l'échangeur au point considéré du milieu

$\lambda$ et $a$ la conductivité et la diffusivité thermique

$p$ la puissance dissipée par l'échangeur par unité de longueur

* le signe du produit de convolution

soit sous forme numérique :

$$
\theta(r, t)=\frac{1}{4 \pi \lambda} \sum_{i} \Delta p_{i} E_{1}\left(\frac{r^{2}}{4 a\left(t-t_{i}\right)}\right)
$$

où $\Delta p_{t}$ est l'incrément de puissance à l'instant $t$

L'instrumentation thermique permet le contrôle des puissances échangées sur sept des dix-neuf échangeurs, la mesure des températures moyennes de ces échangeurs ainsi que dans le sol à proximité de certains d'entre eux.

L'ajustement de la relation (2) sur les données expérimentales permet qualitativement de mieux cerner le domaine de validité du schéma de conduction pure retenue pour décrire les transferts et d'accéder à une estimation quantitative de la conductivité thermique apparente au voisinage des échangeurs. 


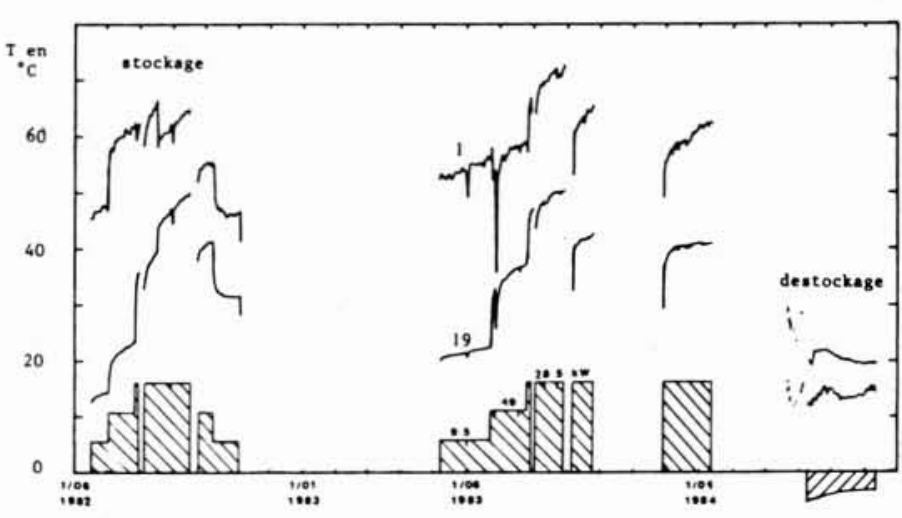

Figure 6. - Programme de chargement et déstockage. Température d'entrée des échangeurs 1 et 19 pendant les phases " actives".

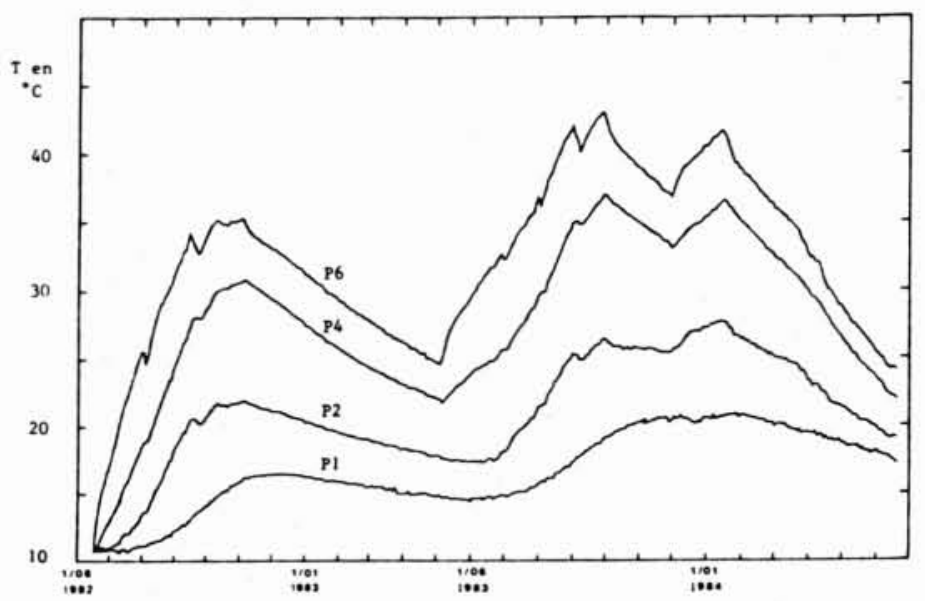

Figure 7. - Evolution de la température du sol en quelques points.

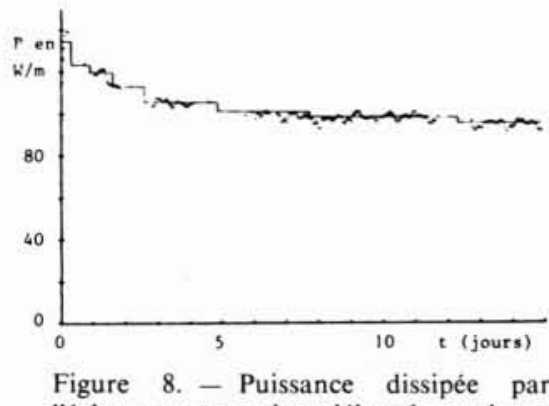

l'échangeur central au début du stockage.

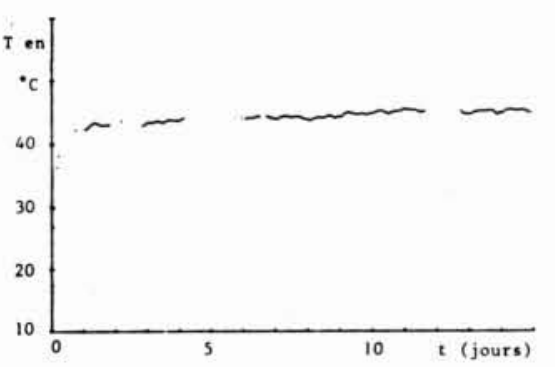

Figure 9. - Température moyenne de l'échangeur central.

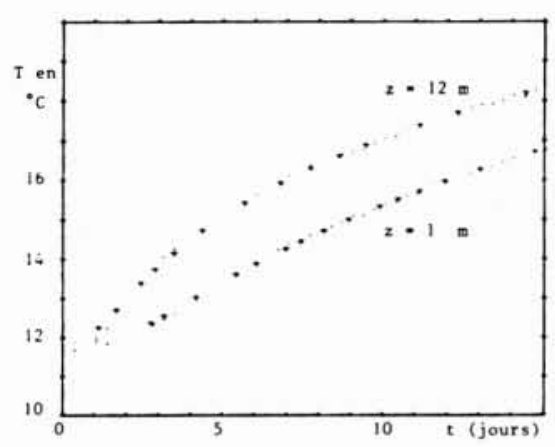

Figure 10. - Température du sol près de l'échangeur central (profil P6).
La figure 8 donne l'allure au cours des deux premières semaines d'injection de la chaleur, de la puissance échangée par l'échangeur central ainsi que la fonction escalier de puissance considérée dans la relation (2). La figure 9 montre l'évolution de la température moyenne de cet échangeur.

La figure 10 représente l'évolution de la température dans le sol à $1 \mathrm{~m}$ et $12 \mathrm{~m}$ de profondeur, au niveau du profil P6 proche de l'échangeur central. Les températures de sol, calculées après estimation des deux paramètres inconnus de la relation (2) $\lambda$ et $r^{2} / a$ ont été portées sur cette figure. L'accord entre les données expérimentales et les points calculés en dépit d'hypothèses de calcul assez fortes, témoigne du fait qu'un modèle purement conductif suffit à décrire les transferts de chaleur dans le milieu.

Les valeurs de conductivité apparente, obtenues à partir des évolutions expérimentales de température des échangeurs E1 et E3 et des profils P6 ou P4 situés à environ $1 \mathrm{~m}$ respectivement de ces échangeurs, sont résumées dans le tableau ci-dessous :

\begin{tabular}{|c|c|c|ccc|}
\hline Echangeur & $\lambda$ en W/(mK) & Profil & $z=1 \mathrm{~m}$ & $z=5 \mathrm{~m}$ & $z=12 \mathrm{~m}$ \\
E1 & 2.3 & P6 & 1.5 & 2.2 & 2.3 \\
E3 & 2.4 & P4 & 1.8 & 2.3 & 2.5 \\
\hline
\end{tabular}

Ces valeurs apparaissent compatibles entre elles : au mois de juin la zone superficielle du terrain n'est pas saturée et la conductivité thermique y est plus modérée, par exemple.

La diffusivité thermique n'est pas accessible. Ce paramètre n'apparaissant que dans le groupe $r^{2} / a$, son estimation nécessiterait la connaissance précise de la distance $r$. 

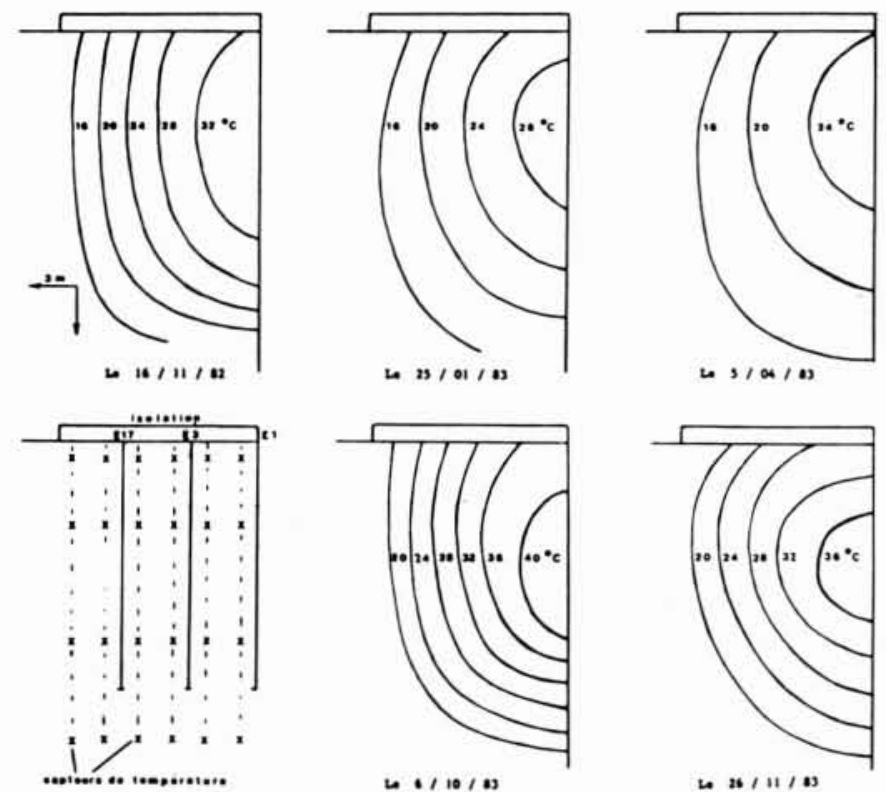

2. $4111 \%$

Figure 11. - Evolution des isothermes dans un plan radial pendant les phases de refroidissement naturel.

Dans le cas des échangeurs, la géométrie est trop complexe. Dans le cas des capteurs correspondant aux profils, un repérage suffisamment précis de la position des cannes de température par rapport aux échangeurs n'a pu être réalisé.

\subsection{Interprétation des phases de refroidissement naturel}

Le champ de température à l'intérieur du stock en période de stockage ou de déstockage est complexe. Ce champ se lisse très vite, lorsqu'on laisse le stock évoluer naturellement sans injection ni extraction de chaleur. Compte tenu de la configuration du stock, on peut alors admettre une diffusion de la chaleur sensiblement radiale. Les 24 thermocouples des profils P1 à P6 permettent un échantillonnage représentatif de la température dans un plan radial, échantillonnage à partir duquel on peut estimer une diffusivité moyenne du milieu de stockage.

L'équation indéfinie de la chaleur s'écrit en effet sous forme intégrale :

$$
\iint_{s} \frac{\partial \theta}{\partial n} d s=\frac{1}{a} \frac{\partial}{\partial t} \iiint_{v} \theta d v
$$

où a est la diffusivité thermique,

$\mathrm{v}$ un volume quelconque,

s la surface limitant ce volume,

$\partial \theta / \partial n$ indique la composante normale à cette surface du gradient de température.
La figure 11 représente les isothermes au cours du premier hiver de fonctionnement du stock puis au cours de la phase de relaxation survenue à l'automne 83, respectivement 2 semaines, 12 semaines et 22 semaines puis 10 et 60 jours après arrêt des injections de la chaleur. On remarque le caractère très régulier des champs de température.

L'application de la relation (3) à un volume cylindrique axé sur l'échangeur central permet d'estimer la diffusivité thermique à environ $810^{-7} \mathrm{~m}^{2} / \mathrm{s}$, quelque soit le temps donc indépendamment du niveau thermique.

\subsection{Mesures à la sonde de conductivité thermique}

Les sondes utilisées sont du type monotige à choc thermique.

Trois séries de mesures ont été réalisées avec ces sondes : la première, au mois de juin 1982 avant la mise en fonctionnement du stock, la seconde à la fin du premier hiver en avril 83 , la troisième au cours de la seconde phase de stockage, en août 83 . Les valeurs de conductivité obtenues sont portées dans le tableau suivant :

\begin{tabular}{|c|c|c|c|c|c|c|c|}
\hline \multicolumn{2}{|c|}{$\begin{array}{l}\text { Profondeur } \\
\text { d'implantation } \\
\text { en m }\end{array}$} & 8 & 8 & 8 & 2 & 1 & 1 \\
\hline $\begin{array}{c}\lambda \text { en } \\
W /(m K)\end{array}$ & $\begin{array}{l}15 / 06 / 82 \\
29 / 04 / 83 \\
19 / 08 / 83\end{array}$ & $\begin{array}{l}2.08 \\
2.17\end{array}$ & $\begin{array}{l}2.2 \\
2.2 \\
2.1\end{array}$ & $\begin{array}{l}2.54 \\
2.47\end{array}$ & $\begin{array}{l}1.53 \\
1.54 \\
1.53\end{array}$ & $\begin{array}{l}1.34 \\
1.7 \\
1.7\end{array}$ & $\begin{array}{l}2.23 \\
1.8\end{array}$ \\
\hline
\end{tabular}

Malgré les pertes d'informations causées par une alimentation stabilisée et une référence froide en température défectueuses, on peut tirer les conclusions suivantes :

- le milieu de stockage est hétérogène et la tranche superficielle du sol est nettement moins conductrice; - une éventuelle dépendance de la conductivité thermique avec la température n'est pas détectable, pour les niveaux thermiques atteints;

- la conductivité à $1 \mathrm{~m}$ semble varier de façon importante au cours du temps, variation liée apparemment à l'état hydrique local (confère les mesures hydriques plus loin), impossible cependant à interpréter en l'absence de mesures complémentaires.

La comparaison des valeurs obtenues avec la sonde et des estimations déduites de l'interprétation des évolutions de température dans le sol montre que pour ce type de terrain, les mesures effectuées avec la sonde dont le rayon d'action est inférieur à $10 \mathrm{~cm}$ peuvent être représentatives de volumes de sol d'étendue supérieure au mètre.

On regrettera l'absence de résultats concernant la diffusivité. Les valeurs jusqu'alors obtenues, voisines de $1.10^{-6} \mathrm{~m}^{2} / \mathrm{s}$, sont en assez bon accord avec l'estimation, à l'échelle du stockage, déduite des phases de refroidissement naturel. Cependant, en raison de l'imprécision entachant les valeurs, une interprétation plus fine des mesures était nécessaire. Validée en laboratoire, une nouvelle méthode n'a pu à cette date être testée sur des mesures in situ. 

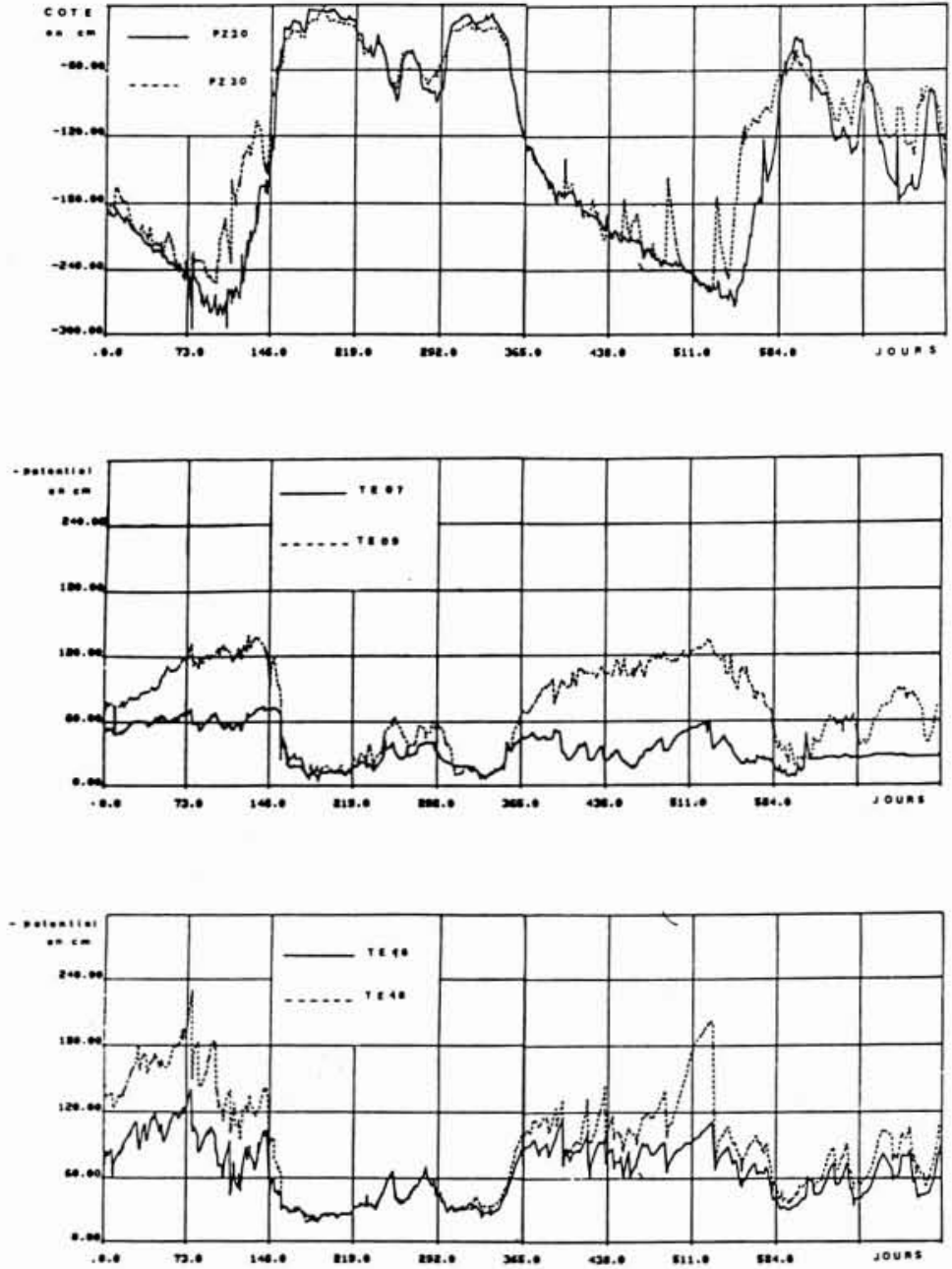

Figure 12. - Variation des niveaux piézométriques.
Figure 13. Evolution des potentiels au centre du stock.

Figure 14. - Evolution des potentiels à la périphérie du stock.

\section{Mesures hydriques}

La figure 3 rappelle l'implantation des différents capteurs destinés à suivre l'évolution de l'état hydrique du stock expérimental : piézomètres, tensiomètres, cellules capacitives, tubes pour la mesure de la teneur en eau par méthode neutronique.

On examinera successivement les mesures piézométriques, la mesure de potentiel dans la zone superficielle temporairement non saturée, et la mesure de teneur en eau.

\subsection{Mesures piézométriques}

La figure 12 présente les variations observées du niveau piézométrique, du 15 juin 1982 au 15 juin 1984, dans les piézomètres $\mathrm{PZ} 20$ et $\mathrm{PZ} 30$ situés respectivement au centre du stock et à sa périphérie.

Les observations effectuées dans le piézomètre PZ 30 sont tout à fait comparables à ce qui est observable en site libre: abaissement lent du niveau piézométrique au printemps et en été, de la surface du sol à environ $3 \mathrm{~m}$ de profondeur puis remontée rapide en automne et début d'hiver. Ce piézomètre est de plus très sensible aux événements météorologiques, les pluies se manifestant par des remontées brutales du niveau piézométrique. Le piézométrique $\mathrm{PZ} 20$ ne se distingue du précédent, outre une moins grande sensibilité aux pluies, que par un retard, très net, de la remontée du niveau phréatique, retard qui reste cependant sans effet sur le niveau hivernal.

\subsection{Potentiels}

Les figures 13 et 14 présentent les variations de potentiel mesurées à $0,50 \mathrm{~m}$ et $1,50 \mathrm{~m}$ de profondeur, à proximité du centre du stock (tensiomètres TE 07 et TE 09) et à la périphérie (tensiomètres TE 16 et TE 18), pendant la même période. 
Les remarques suivantes peuvent être faites sur ces figures :

- Au cours de la seconde année, aussi bien au centre du stock qu'à la périphérie les potentiels varient de façon analogue à ce qui a été observé au cours de la première année.

- En période de "basses eaux " le potentiel à $1,50 \mathrm{~m}$ de profondeur est toujours inférieur au potentiel à $0,50 \mathrm{~m}$ de profondeur, ce qui témoigne d'une infiltration verticale. Ce point est confirmé par la comparaison des potentiels aux niveaux phréatiques au même moment.

- Les potentiels mesurés à l'extérieur du stock atteignent des valeurs sensiblement inférieures à celles observées au centre du stock, avec des variations à court terme de bien plus grande ampleur.

\subsection{Teneurs en eau}

Deux techniques de mesure avaient été retenues: la méthode capacitive et la méthode neutronique.

Du fait de la faible résistivité des eaux d'infiltration et de phénomènes pédologiques qui n'ont pas été élucidés, les données fournies par les sondes capacitives se sont révélées inexploitables. $\mathrm{La}$ méthode neutronique a par contre permis de suivre l'évolution de l'état hydrique du stock en 5 profils. Les résultats obtenus ne sont pas illustrés ici : ils indiquent des variations très modérés de la teneur en eau au delà de $1 \mathrm{~m}$ de profondeur, les variations les plus importantes étant observées vers $35 \mathrm{~cm}$ de profondeur. On observe notamment aucun assèchement de la zone chaude du stock.

L'ensemble des observations effectuées, alliées aux connaissances préalables des conditions hydrogéologiques locales, conduisent à la représentation suivante du fonctionnement hydrique du stock :

L'imperméabilisation, due à l'isolation thermique superficielle du stock, perturbe le régime hydrogéologique local. Cette perturbation se traduit par une moins grande variation de l'état hydrique du sol qu'en site libre, l'écran imperméable superficiel s'opposant autant à l'assèchement estival du sol qu'à sa réhumidification hivernale. Une couche superficielle plus perméable, de moins d'un mètre d'épaisseur, dans laquelle est observée la plus grande variation d'état hydrique, permet cependant, avec un certain retard, la réalimentation hivernale du stock.

Les effets thermiques éventuels, dans ce contexte, ne sont pas observables.

\section{Conclusion}

Les résultats de l'interprétation des données, à ce jour réalisée, amènent aux conclusions suivantes :

Le milieu de stockage peut être correctement représenté par un matériau purement conductif. Des phénomènes de thermomigration dans la couche superficielle saturée ne sont pas détectables; ce point est à rapprocher des observations effectuées par Saix et al. [4].

La mesure en place des caractéristiques thermocinétiques des sols, mesure qui s'est avérée représentative du milieu dans cette expérimentation, peut-être obtenue à l'aide d'une sonde monotige à choc thermique.

Malgré un rendement faible de cette installation, qualitativement en accord avec les prévisions, le stockage de chaleur par conduction dans le sol, à des niveaux de température compatibles avec une utilisation directe de l'énergie déstockée pour le chauffage des locaux par exemple, semble possible, à l'échelle de l'habitat collectif toutefois.

\section{Références}

[1] G. Leroy et R. SANejouAnd. - Stockage intersaisonnier de chaleur dans le sol à basse température. Congrès sur le stockage thermique et sa modélisation. La Baule, mai 1982. Voir la R.G.T., février 1983.

[2] G. LEROY. - Etude d'un stockage intersaisonnier de chaleur dans le sol par forage et échangeurs tubulaires équirépartis. Mémoire de thèse de doctorat de $3^{\circ}$ cycle, Paris VI, décembre 1982.
[3] R. Sanejouand, G. Leroy et J.P. Bardon. - Seasonal heat storage by conduction in soil. International conference on subsurface heat storage in theory and practice. Stockholm, 6-8 juin 1983.

[4] C. Saix, G. Della Vallée, J.C. Benet, P. Jouana. - Physical model for the study of mass and energy transfers in the non saturated layer of soil above a heat storage zone. International conference on subsurface heat storage in theory and practice. Stockholm, 6-8 juin 1983. 
M. FIRDAOUSS, - Alors que les températures initiales sont presque identiques quelle que soit la profondeur, pour quelle raison la température à $1 \mathrm{~m}$ de profondeur évolue-t-elle moins rapidement que la température à $12 \mathrm{~m}$ de profondeur. Est-ce dû à la distance de l'échangeur ou à des caractéristiques thermiques différentes voire à la teneur en eau ?

M. LEROY. - Ceci résulte essentiellement dans ce cas du fait que la conductivité thermique du sol est plus faible en surface qu'en profondeur : la puissance cédée localement par l'échangeur varie au premier ordre comme la conductivité. L'évolution de la température en surface est donc moins rapide.

M. GAUDET. - Dans les trois premières communications on a toujours considéré un milieu de stockage favorable dont l'humidité ne varie pas en cours d'année ou à proximité des échangeurs. Dans les sols sableux les importantes variations saisonnières de la saturation rendraient nécessaire la prise en compte de paramètres thermiques variables.

M. LAUNAY. - Avez-vous une idée du coût des forages et quelle est l'influence de la position des capteurs de température sur la précision du calcul des paramètres thermoactifs?

M. MEROY. - Une imprécision sur la position du capteur n'influence pas la mesure de la conductivité, mais a une forte influence sur la valeur de la diffusivité.
Dans cette expérience le coût des forages a été approximativement de $100 \mathrm{~F}$. par mètre sachant que ce coût inclut celui du déplacement d'une machine.

M. BANAL. - J'avais retenu de la communication de $\mathrm{M}$. MENJOZ une impression favorable, mais les deux autres communications me paraissent un peu inquiétantes au point de vue performances. Est-ce fondé ou pas ?

M. GAUDET. - Dans la première étude les pertes thermiques du terrain correspondent à un gain d'énergie pour le fluide des échangeurs, alors que dans les deux cas suivants le terrain joue le rôle d'un stockage thermique dont les pertes thermiques sont d'autant plus importantes que l'écart entre les températures du stock et de la surface du sol est grand et que la taille du stockage est réduite.

M. LEROY. - Il existe un temps de mise en régime relié à la constante de temps du système. Les pertes thermiques initiales ne sont pas totalement défavorables dans la mesure où elles permettent d'élever le niveau thermique du milieu environnant et d'atteindre un régime permanent au cours duquel les pertes thermiques sont moindres.

M. SourieaU. - On rencontre le même problème dans le stockage du méthane liquéfié dans des cavités souterraines. Il faut attendre un nombre important de cycles pour atteindre un régime à peu près asymptotique.

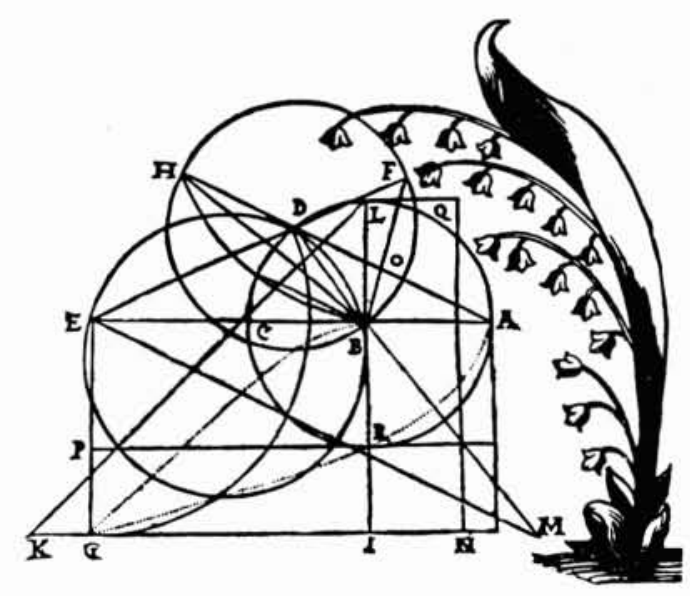




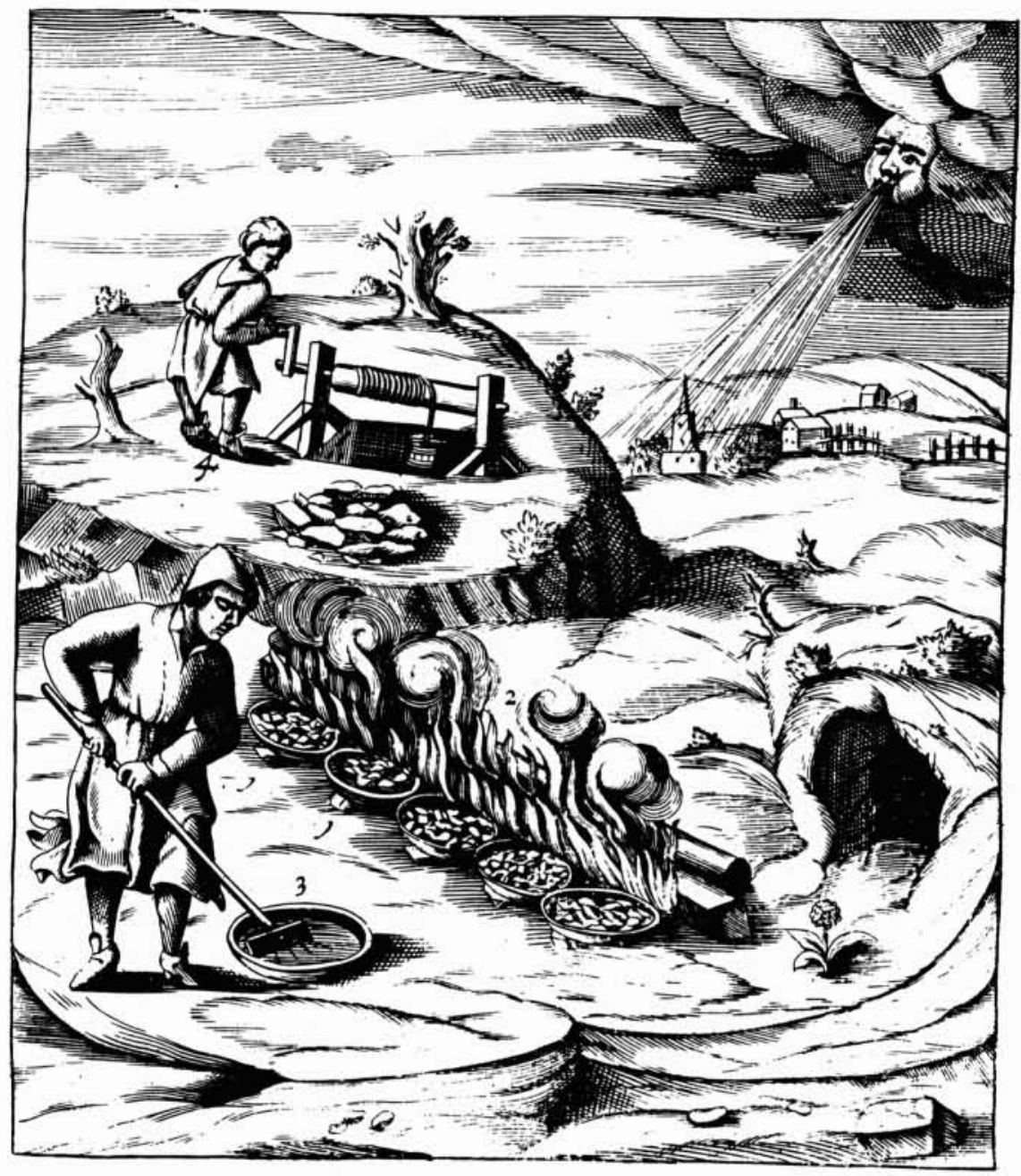

Deciphered.

1. The little Iron Pans for Spelter or Wifmet Oar.

2. The fire of vvood for them.

3. Melted Spelter $t$ bat is to be made clean in tbe iron $\mathrm{Pan}$, and the mork.man that tends it.

4. He tbat draws tbe Oar out of tbe Mine. 